\title{
To self, or not to self... A review of outcrossing and pollen-mediated gene flow in neotropical trees
}

\author{
M Ward ${ }^{1}$, CW Dick ${ }^{2,4}$, R Gribel ${ }^{3}$ and AJ Lowe ${ }^{1}$ \\ ${ }^{1}$ School of Integrative Biology, University of Queensland, Brisbane, Queensland 4072, Australia; ${ }^{2}$ Smithsonian Tropical Research \\ Institute, Apartado 0843-03092, Balboa, Republic of Panama; ${ }^{3}$ Instituto Nacional de Pesquisas da Amazonia (INPA), Avenida André \\ Araújo 2936, Manaus, Amazonas, Brazil
}

\begin{abstract}
Despite the typically low population densities and animalmediated pollination of tropical forest trees, outcrossing and long-distance pollen dispersal are the norm. We reviewed the genetic literature on mating systems and pollen dispersal for neotropical trees to identify the ecological and phylogenetic correlates. The 36 studies surveyed found $>90 \%$ outcrossed mating for 45 hermaphroditic or monoecious species. Selffertilization rates varied inversely with population density and showed phylogenetic and geographic trends. The few direct measures of pollen flow ( $N=11$ studies) suggest that pollen dispersal is widespread among low-density tropical trees,
\end{abstract}

ranging from a mean of $200 \mathrm{~m}$ to over $19 \mathrm{~km}$ for species pollinated by small insects or bats. Future research needs to examine (1) the effect of inbreeding depression on observed outcrossing rates, (2) pollen dispersal in a wide range of pollination syndromes and ecological classes, (3) and the range of variation of mating system expression at different hierarchical levels, including individual, seasonal, population, ecological, landscape and range wide.

Heredity (2005) 95, 246-254. doi:10.1038/sj.hdy.6800712; published online 10 August 2005

Keywords: mating system; gene flow; neotropical trees; pollination; pollen dispersal

\section{Introduction}

Mating systems, the genetic relatedness and patterns of pairings between gametes (as distinct from breeding systems, the morphological and physiological characteristics of pairing; Neal and Anderson, 2005), play a central role in evolutionary theories of the origin of tree species richness in the tropics (Bawa, 1992). In species-rich tropical rainforests, the population density of adult trees of a given species is generally low and thus distances between the crowns of flowering conspecifics will be large (Hubbell and Foster, 1983). This observation originally led botanists to predict that most tropical tree species should be highly self-fertilizing or inbred (eg Corner, 1954; Baker, 1959; Fedorov, 1966), under the premise that animal pollinators are unable to move among widely spaced conspecifics. Over the last 30 years, this early view of tropical pollinators and the mating systems of tropical tree species has been completely revised. Community-level studies of breeding systems in tropical trees revealed high levels of dioecey $(>20 \%)$, and cross-pollination studies provided evidence of self-incompatibility in hermaphroditic or monoecious species (Bawa et al, 1985). Genetic markerbased analyses of mating system have tended to confirm these field studies, revealing high rates of outcrossing and long-distance pollen dispersal for a range of

Correspondence: $M$ Ward, School of Integrative Biology, University of Queensland, St Lucia, Brisbane, Queensland 4072, Australia.

E-mail: s4087128@student.uq.edu.au

${ }^{4}$ Current address: Department of Ecology and Evolution and Herbarium, University of Michigan, Ann Arbor, MI 48109-1048, USA

Received 1 March 2005; accepted 13 June 2005; published online 10 August 2005 pollination syndromes (eg Boshier et al, 1995a,b; Stacy et al, 1996; Loveless et al, 1998; Nason et al, 1998).

Genetic marker-based studies have also revealed that outcrossing rate and pollen dispersal distances are sensitive to ecological factors, and show variation over both spatial and temporal scales (Nason and Hamrick, 1997). For example, factors such as population density and pollinator abundance and composition change over the range of a species, have a concomitant impact on outcrossing rate and pollen-mediated dispersal at a landscape scale (Franceschinelli and Bawa, 2000; Dick et al, 2003; Degen et al, 2004). Mating system analyses also indicate the potential for variation in the relative rates of selfing and outcrossing at a micro-scale, where variation occurs among individuals within populations, among populations over years and from one flowering event to another (Murawski and Hamrick, 1991; Nason and Hamrick, 1997). Finally, phylogenetic constraints on floral morphology and self-compatibility system are also expected to influence the observed outcrossing rates and patterns of pollen dispersal (Gribel et al, 1999).

This review examines studies that use molecular genetic techniques to quantify mating systems and pollen-mediated gene dispersal in neotropical tree species. The review is limited to the neotropics, as the authors are familiar with the study species in this region. However, given the pantropical distribution of most tropical tree families and many genera (Pennington and Dick, 2004), our conclusions should have broad geographic application. Our discussion of pollen dispersal is focussed on undisturbed populations, as Lowe et al (2005) reviews studies in fragmented habitats. Our objectives are to (1) examine the relationship between 
the multilocus outcrossing rate $\left(t_{\mathrm{m}}\right)$ and phylogenetic and ecological factors, such as population density, pollination syndrome and habitat change, and (2) determine the distances over which pollen is dispersed among low-density, animal-pollinated trees in speciesrich lowland neotropical forests.

\section{Review of the literature}

A total of 36 case studies encompassing 45 neotropical tree species were surveyed. For 29 of these case studies, a multilocus mixed-mating model (eg Ritland and Jain, 1981; Ritland, 1986) was used to determine the mating system. Henceforth, 'mating system' is used synonymously with the outcrossing rate. Some estimate of pollen dispersal was made in 10 studies. Allozymes were employed in 22 case studies, whereas microsatellites were utilized in 14 case studies. The results of the literature survey are summarized in Table 1.

\section{Correlates of mating system}

Phylogeny: As the anatomical and biochemical aspects of self-incompatibility systems may be phylogenetically constrained (Gribel et al, 1999), we looked for phylogenetic association with the mating system. The taxonomic breadth encompassed by the relevant literature presently includes 15 plant families (Table 1), with an average of 2.9 species examined per family.

It is noteworthy that species in the Malvaceae (subfamily Bombacoideae; former Bombacaceae) frequently exhibit outcrossing rates lower than $80 \%$ (mixed-mating systems). Mixed-mating systems were found in Pachira quinata (Fuchs et al, 2003), Cavanillesia platanifolia (Murawski and Hamrick, 1992b), Ceiba pentandra (Murawski et al, 1990; Murawski and Hamrick, 1991, 1992a; Gribel et al, 1999; Lobo et al, 2005) and Pseudobombax munguba (Gribel and Gibbs, 2002). This pattern may apply more generally to trees in the order Malvales, which includes the Sterculiaceae, Ebenaceae and Tiliaceae. Self-fertilization rates higher than 50\% have been documented in Shorea and Stemonoporus of the Dipterocarpaceae (Murawski and Bawa, 1994; Murawski et al, 1994), which is the dominant family of tropical trees in Asia, also in the Malvales. The dominant tree family of Neotropical forests - the legume family Fabaceae - also exhibits patterns of mixed mating, with outcrossing rates as low as 0.54 in the pioneer legume Senna multijuga (Ribeiro and Lovato, 2004), 0.42 in Dicorynia guianensis (Latouche-Hallé et al, 2004), <0.50 in Platypodium elegans (Hufford and Hamrick, 2003) and 0.63 in Dinizia excelsa (Dick et al, 2003).

Published mating system studies do not encompass the taxonomic richness of tropical forests. We found no mating system studies for some of the most species-rich neotropical tree families, including the Sapotaceae, Annonaceae, Myrtaceae, Chrysobalanaceae and Burseraceae. In controlled-pollination studies, however, Bawa et al (1985) report successful self-pollination in the Annonaceae. In the absence of genetic analysis, however, it is not possible to distinguish between self-fertilization and apomixis in such studies. Apomixis, the parthenogenic production of seed, can be induced by pollen, and has been documented in the Malvacean tree genera Pachira (Baker, 1960), Bombacopsis (Duncan, 1970) and Eriotheca (Oliveira et al, 1992), and it has also been documented in the Dipterocarpaceae (Kaur et al, 1978). Apomixis may have an important role in the breeding structure of some populations. In a fascinating Malaysian study of a species in the pantropical genus Garcinia (G. scortechinii), Thomas (1997) found an entirely female population that persisted solely through apomixis.

High outcrossing rates result from self-incompatibility, and may be phylogenetically constrained, but inbreeding depression at the embryo stage can also produce largely outcrossed offspring (Seavey and Bawa, 1986). In order to distinguish between the effects of self-incompatibility and inbreeding depression, Hufford and Hamrick (2003) documented the change in outcrossing rate in P. elegans (Fabaceae) at three early life stages: aborted embryos, mature seeds and seedlings. The outcrossing rate increased across each life stage $\left(t_{\mathrm{m}}=0.79,0.82\right.$ and $0.91)$, suggesting that inbreeding depression may explain high outcrossing observed in seedlings, the stage at which many genetic marker studies of mating system are carried out. Several neotropical trees also have lateacting self-incompatibility mechanisms (eg Tabebuia; Bittencourt and Semir, 2005), and so a genetic estimation of outcrossing rate would be strongly influenced by the developmental stage at which seeds are assessed.

Individual, population, range and landscape variation: Individual outcrossing rates can vary widely. Murawski and Hamrick (1992a) reported outcrossing rates in C. pentandra (Malvaceae) ranging from complete selffertilization to complete outcrossing. This variation was explained by asynchrony in flowering times and nonrandom foraging behaviour of the bat pollinators. Similar results for C. pentandra reported by Gribel (in Wilson et al, 2001) showed the percentage of selfed seeds to range from zero to $97.8 \%$ for individual trees. Multilocus outcrossing rates for 25 individual trees varied from 0.38 to 1.00 in an Amazonian population of Swietenia macrophylla (Meliaceae), although 23 individuals were predominantly or completely outcrossing (Lemes, 2000). Similarly, Latouche-Hallé et al (2004) observed marked variation in outcrossing rates among individuals of D. guianensis (Fabaceae), likely due to asynchrony in flowering times. Rocha and Aguilar (2001) report spatial and temporal variation in outcrossing rates in Enterolobium cyclocarpum over two consecutive years. Despite these multiple sources of individual variation in outcrossing rates, there are some clear ecological correlates of mating system variation.

Murawski and Hamrick (1991) examined the relationship between the density of flowering individuals in a population and its outcrossing rate in nine neotropical tree species, and found that three representatives from the Bombacoideae (Malvaceae) exhibited a correlation between outcrossing rate and flowering tree density. Additionally, Murawski and Hamrick (1992b) reported low outcrossing rates in a low-density population of C. platanifolia $\left(t_{\mathrm{m}}=0.213\right)$ compared to a high-density population $\left(t_{\mathrm{m}}=0.661\right)$. Murawski and Hamrick (1992b) and Nason and Hamrick (1997) report this pattern for other species.

There can be a geographic or historical component of mating system variation. C. pentandra has a neotropical origin, but has become established in Africa via oceanic dispersal at least 14000 years before present (Dick, unpublished data). While neotropical populations dis- 
Table 1 Biological, mating system and pollen-mediated gene flow characteristics of case-study species

\begin{tabular}{|c|c|c|c|c|c|c|c|c|c|}
\hline Family & Species & Region & Life history & Pollination system & Genetic marker & Sampling design & $\mathrm{t}_{m}$ & Gene flow & Reference \\
\hline Anacardiaceae & Spondias mombin & Panama & Canopy & $\begin{array}{l}\text { Variety of small } \\
\text { insects }\end{array}$ & Allozymes & $\begin{array}{l}\text { Leaves and progeny } \\
\text { from a } 84 \text { ha } \\
\text { population }\end{array}$ & $\begin{array}{l}t_{\mathrm{m}}=0.989 \pm 0.163 \text { and } \\
1.304 \pm 0.108\end{array}$ & $\begin{array}{l}<6 \% \text { pollen } \\
\text { movement } \\
\text { over } 300 \mathrm{~m}\end{array}$ & Stacy et al (1996) \\
\hline Apocynaceae & $\begin{array}{l}\text { Stemmadenia } \\
\text { donnell-smithii }\end{array}$ & Costa Rica & Subcanopy & $\begin{array}{l}\text { Euglossine bees, } \\
\text { butterflies }\end{array}$ & Allozymes & $\begin{array}{l}\text { Progeny from a } \\
\text { single disturbed } \\
\text { patch }\end{array}$ & $t_{\mathrm{m}}=0.896 \pm 0.107$ & & James et al (1998) \\
\hline Arecaceae & $\begin{array}{l}\text { Astrocaryum } \\
\text { mexicanum }\end{array}$ & Mexico & Understory & Small beetles & Allozymes & $\begin{array}{l}\text { Progeny from four } \\
\text { plots, collections } \\
\text { from three diferent } \\
\text { years }\end{array}$ & $\begin{array}{l}t_{\mathrm{m}}=\text { from } 0.933 \pm 0.174 \\
\text { to } 1.050 \pm 0.066\end{array}$ & & $\begin{array}{l}\text { Eguiarte et al } \\
\text { (1992) }\end{array}$ \\
\hline Arecaceae & Euterpe edulis & Brazil & Canopy & Small bees, wind? & Microsatellites & $\begin{array}{l}\text { Adults, juveniles } \\
\text { and seedlings from } \\
\text { two plots }\end{array}$ & $\begin{array}{l}t_{\mathrm{m}}=0.90 \pm 0.04 \text { and } \\
0.98 \pm 0.02, \text { no } \\
\text { biparental inbreeding }\end{array}$ & & $\begin{array}{l}\text { Gaiotto et al } \\
\text { (2003) }\end{array}$ \\
\hline Bignoniaceae & Jacaranda copaia & Costa Rica & $\begin{array}{l}\text { Canopy, } \\
\text { pioneer }\end{array}$ & $\begin{array}{l}\text { Medium to large } \\
\text { bees }\end{array}$ & Allozymes & $\begin{array}{l}\text { Progeny from two } \\
\text { disturbed patches } \\
1 \mathrm{~km} \text { apart }\end{array}$ & $t_{\mathrm{m}}=0.943 \pm 0.044$ & & James et al (1998) \\
\hline Boraginaceae & Cordia alliodora & Costa Rica & Canopy & & Allozymes & $\begin{array}{l}\text { Progeny from a } \\
\text { single stand }\end{array}$ & $\mathrm{t}=0.904$ to 0.978 & $\begin{array}{l}\text { Overall } \\
\text { extensive gene } \\
\text { flow; distances } \\
\text { up to } 280 \mathrm{~m}\end{array}$ & $\begin{array}{l}\text { Boshier et al } \\
(1995 a, b)\end{array}$ \\
\hline Caryocaraceae & $\begin{array}{l}\text { Caryocar } \\
\text { brasiliense }\end{array}$ & Brazil & Canopy & Bats & Microsatellites & $\begin{array}{l}\text { Leaves and progeny } \\
\text { from four } \\
\text { populations }\end{array}$ & $\begin{array}{l}t_{m}=1.00, \text { high } \\
\text { biparental inbreeding }\end{array}$ & & $\begin{array}{l}\text { Collevatti et al } \\
\text { (2001) }\end{array}$ \\
\hline Clusiaceae & $\begin{array}{l}\text { Calophyllum } \\
\text { longifolium }\end{array}$ & Panama & Canopy & $\begin{array}{l}\text { Variety of small } \\
\text { insects }\end{array}$ & Allozymes & $\begin{array}{l}\text { Leaves and progeny } \\
\text { from a } 84 \text { ha } \\
\text { population }\end{array}$ & $\begin{array}{l}t_{\mathrm{m}}=1.030 \pm 0.085 \text { and } \\
1.031 \pm 0.035\end{array}$ & $\begin{array}{l}62 \% \text { pollen } \\
\text { moved more } \\
\text { than } 210 \mathrm{~m}\end{array}$ & Stacy et al (1996) \\
\hline Clusiaceae & $\begin{array}{l}\text { Symphonia } \\
\text { globulifera }\end{array}$ & Costa Rica & Canopy & Hummingbirds & Microsatellites & $\begin{array}{l}\text { Progeny arrays from } \\
\text { forest fragments and } \\
\text { pastures }\end{array}$ & $\begin{array}{l}t_{\mathrm{m}}=0.91 \text { and } 0.89 \mathrm{in} \\
\text { continuous forest and } \\
\text { forest fragments; } \\
t_{\mathrm{m}}=0.74 \text { in pasture } \\
\text { trees }\end{array}$ & & $\begin{array}{l}\text { Aldrich and } \\
\text { Hamrick (1998) }\end{array}$ \\
\hline Clusiaceae & $\begin{array}{l}\text { Symphonia } \\
\text { globulifera }\end{array}$ & French Guiana & Canopy & Hummingbirds & Microsatellites & $\begin{array}{l}\text { Cambium and } \\
\text { progeny from } \\
\text { three plots within } \\
\text { a single site }\end{array}$ & $\begin{array}{l}t_{\mathrm{m}}=0.92 ; \text { high } \\
\text { biparental inbreeding }\end{array}$ & $\begin{array}{l}\text { Mean pollen } \\
\text { dispersal } \\
\text { between } 27 \\
\text { and } 53 \mathrm{~m}\end{array}$ & $\begin{array}{l}\text { Degen et al } \\
\text { (2004) }\end{array}$ \\
\hline Lauraceae & $\begin{array}{l}\text { Beilschmedia } \\
\text { pendula }\end{array}$ & Panama & & Possibly bees & Allozymes & $\begin{array}{l}\text { Progeny from a } \\
\text { single plot }\end{array}$ & $t_{\mathrm{m}}=0.918 \pm 0.058$ & & $\begin{array}{l}\text { Murawski and } \\
\text { Hamrick (1991) }\end{array}$ \\
\hline Lauraceae & Ocotea tenera & Costa Rica & & $\begin{array}{l}\text { Small bees, } \\
\text { flies and wasps }\end{array}$ & Allozymes & $\begin{array}{l}\text { Leaves and progeny } \\
\text { from a } 16 \mathrm{~km}^{2} \\
\text { population }\end{array}$ & $t_{\mathrm{m}}=0.846-0.965$ & & $\begin{array}{l}\text { Gibson and } \\
\text { Wheelwright } \\
\text { (1996) }\end{array}$ \\
\hline Lecythidaceae & $\begin{array}{l}\text { Bertholletia } \\
\text { excelsa }\end{array}$ & $\begin{array}{l}\text { Brazilian } \\
\text { Amazon }\end{array}$ & Canopy & Large bees & Allozymes & Progeny from a & $t_{\mathrm{m}}=0.85 \pm 0.03$ & & O'Malley et al \\
\hline $\begin{array}{l}\text { Leguminosae } \\
\text { (Caesalpiniaceae) }\end{array}$ & Dinizia excelsa & $\begin{array}{l}\text { Amazonas, } \\
\text { Braziil }\end{array}$ & $\begin{array}{l}\text { Emergent, } \\
\text { fast-growing }\end{array}$ & $\begin{array}{l}\text { Small insects, } \\
\text { stingless bees, } \\
\text { Apis mellifera in } \\
\text { disturbed habitats }\end{array}$ & Microsatellites & $\begin{array}{l}\text { Multiple progeny } \\
\text { arrays across } \\
\text { fragmented and } \\
\text { continuous forest } \\
\text { landscape }\end{array}$ & $\begin{array}{l}t_{\mathrm{m}}=0.875 \pm 0.049 \text { for } \\
\text { undisturbed forest; } \\
t_{\mathrm{m}}=0.848 \pm 0.044 \text { in } \\
\text { forest fragments and } \\
\text { pasture }\end{array}$ & $\begin{array}{l}\text { Mean pollen } \\
\text { dispersal of } \\
1509 \mathrm{~m} \text { in forest } \\
\text { fragments with } \\
\text { Apis pollination; } \\
212 \mathrm{~m} \text { in un- } \\
\text { disturbed forest }\end{array}$ & Dick et al (2003) \\
\hline
\end{tabular}


Table 1 Continued

\begin{tabular}{|c|c|c|c|c|c|c|c|c|c|}
\hline Family & Species & Region & Life history & Pollination system & Genetic marker & Sampling design & $\mathrm{t}_{m}$ & Gene flow & Reference \\
\hline $\begin{array}{l}\text { Leguminosae } \\
\text { (Caesalpiniaceae) }\end{array}$ & $\begin{array}{l}\text { Vouacapoua } \\
\text { americana }\end{array}$ & French Guiana & Canopy & $\begin{array}{l}\text { Small bees and } \\
\text { thrips }\end{array}$ & Microsatellites & $\begin{array}{l}\text { Progeny from two } \\
\text { stands }\end{array}$ & $5 \%$ selfing & $\begin{array}{l}\text { Short-distance } \\
\text { pollen } \\
\text { dispersal up } \\
\text { to } 100 \mathrm{~m}\end{array}$ & $\begin{array}{l}\text { Dutech et al } \\
\text { (2002) }\end{array}$ \\
\hline $\begin{array}{l}\text { Leguminosae } \\
\text { (Fabaceae) }\end{array}$ & $\begin{array}{l}\text { Dicorynia } \\
\text { guianensis }\end{array}$ & French Guiana & Canopy & Large bees & Microsatellites & $\begin{array}{l}\text { Cambium and } \\
\text { progeny from a } \\
40 \text { ha population }\end{array}$ & $t_{\mathrm{m}}=0.89 \pm 0.044$ & $\begin{array}{l}62 \% \text { of pollen } \\
\text { from outside } \\
\text { site }\end{array}$ & $\begin{array}{l}\text { Latouche-Hallé } \\
\text { et al (2004) }\end{array}$ \\
\hline $\begin{array}{l}\text { Leguminosae } \\
\text { (Fabaceae) }\end{array}$ & $\begin{array}{l}\text { Gliricidia } \\
\text { sepium }\end{array}$ & Guatemala & Pioneer & Large bees & Microsatellites & $\begin{array}{l}\text { Adults and progeny } \\
\text { from a single plot }\end{array}$ & & $\begin{array}{l}6.1 \% \text { of pollen } \\
\text { move more than } \\
75 \mathrm{~m} \text {, longest } \\
\text { dispersal } 275 \mathrm{~m}\end{array}$ & $\begin{array}{l}\text { Dawson et al } \\
\text { (1997) }\end{array}$ \\
\hline $\begin{array}{l}\text { Leguminosae } \\
\text { (Fabaceae) }\end{array}$ & $\begin{array}{l}\text { Platypodium } \\
\text { elegans }\end{array}$ & Panama & Canopy & Bees & Allozymes & $\begin{array}{l}\text { Progeny from a } \\
\text { single plot }\end{array}$ & $\begin{array}{l}t_{\mathrm{m}}=0.924 \pm 0.043 \\
\text { and } 0.898 \pm 0.043\end{array}$ & & $\begin{array}{l}\text { Murawski and } \\
\text { Hamrick (1991) }\end{array}$ \\
\hline $\begin{array}{l}\text { Leguminosae } \\
\text { (Fabaceae) }\end{array}$ & $\begin{array}{l}\text { Platypodium } \\
\text { elegans }\end{array}$ & Panama & Canopy & Bees & Microsatellites & $\begin{array}{l}\text { Aborted fruits, } \\
\text { mature fruits and } \\
\text { germinated } \\
\text { seedlings }\end{array}$ & $\begin{array}{l}t_{\mathrm{m}}=0.79 \text { (aborted } \\
\text { fruit), } 0.82 \text { (mature } \\
\text { seeds) and } 0.91 \\
\text { (seedlings) }\end{array}$ & & $\begin{array}{l}\text { Hufford and } \\
\text { Hamrick (2003) }\end{array}$ \\
\hline $\begin{array}{l}\text { Leguminosae } \\
\text { (Fabaceae) }\end{array}$ & Senna multijuga & Brazil & Pioneer & Bees & Allozymes & $\begin{array}{l}\text { Progeny from two } \\
\text { populations }\end{array}$ & $\begin{array}{l}t_{\mathrm{m}}=0.540 \pm 0.090 \\
\text { and } 0.838 \pm 0.068\end{array}$ & & $\begin{array}{l}\text { Ribeiro and } \\
\text { Lovato (2004) }\end{array}$ \\
\hline $\begin{array}{l}\text { Leguminosae } \\
\text { (Fabaceae) }\end{array}$ & $\begin{array}{l}\text { Tachigalia } \\
\text { versicolor }\end{array}$ & Panama & $\begin{array}{l}\text { Canopy, } \\
\text { monocarpic }\end{array}$ & & Allozymes & $\begin{array}{l}\text { Progeny from six } \\
\text { populations }\end{array}$ & $\begin{array}{l}t_{\mathrm{m}}=0.998 ; \text { no } \\
\text { biparental inbreeding }\end{array}$ & $\begin{array}{l}\text { Large } \\
\text { amounts of } \\
\text { pollen } \\
\text { migration }\end{array}$ & $\begin{array}{l}\text { Loveless et al } \\
\text { (1998) }\end{array}$ \\
\hline $\begin{array}{l}\text { Leguminosae } \\
\text { (Fabaceae) }\end{array}$ & $\begin{array}{l}\text { Tachigalia } \\
\text { versicolor }\end{array}$ & Panama & $\begin{array}{l}\text { Canopy, } \\
\text { monocarpic }\end{array}$ & Bees & Allozymes & $\begin{array}{l}\text { Progeny from a } \\
\text { single plot }\end{array}$ & $t_{\mathrm{m}}=0.937 \pm 0.044$ & & $\begin{array}{l}\text { Murawski and } \\
\text { Hamrick (1991) }\end{array}$ \\
\hline $\begin{array}{l}\text { Leguminosae } \\
\text { (Mimosaceae) }\end{array}$ & $\begin{array}{l}\text { Enterolobium } \\
\text { cyclocarpum }\end{array}$ & Costa Rica & Canopy & $\begin{array}{l}\text { Nocturnal insects, } \\
\text { including moths } \\
\text { and beetles }\end{array}$ & Allozymes & $\begin{array}{l}\text { Progeny from four } \\
\text { populations }\end{array}$ & $\begin{array}{l}t_{\mathrm{m}}=0.812 \text { to } 0.913 ; \\
\text { low biparental } \\
\text { inbreeding } t_{\mathrm{m}}- \\
t_{\mathrm{s}}=0.058 \text { to } 0.079\end{array}$ & & $\begin{array}{l}\text { Rocha and } \\
\text { Aguilar (2001) }\end{array}$ \\
\hline $\begin{array}{l}\text { Leguminosae } \\
\text { (Mimosaceae) }\end{array}$ & $\begin{array}{l}\text { Pithecellobium } \\
\text { elegans }\end{array}$ & Costa Rica & Emergent & & Allozymes & $\begin{array}{l}\text { Progeny from a } \\
150 \text { ha population }\end{array}$ & $t_{\mathrm{m}}=0.970$ and 0.986 & & Hall et al (1996) \\
\hline $\begin{array}{l}\text { Leguminosea } \\
\text { (Mimosaceae) }\end{array}$ & $\begin{array}{l}\text { Pithecellobium } \\
\text { pedicellare }\end{array}$ & Costa Rica & $\begin{array}{l}\text { Canopy, gap- } \\
\text { colonizing }\end{array}$ & & Allozymes & $\begin{array}{l}\text { Progeny from a } \\
600 \text { ha population }\end{array}$ & $t_{\mathrm{m}}=0.951 \pm 0.021$ & & $\begin{array}{l}\text { O'Malley and } \\
\text { Bawa (1987) }\end{array}$ \\
\hline $\begin{array}{l}\text { Malvaceae } \\
\text { (Bombacoideae) }\end{array}$ & $\begin{array}{l}\text { Cavanillesia } \\
\text { platanifolia }\end{array}$ & Panama & $\begin{array}{l}\text { Canopy, gap- } \\
\text { specialist }\end{array}$ & $\begin{array}{l}\text { Hawk moths, } \\
\text { monkeys, bats }\end{array}$ & Allozymes & $\begin{array}{l}\text { Progeny from a } \\
\text { single plot }\end{array}$ & $t_{\mathrm{m}}=0.213 \pm 0.052$ & & $\begin{array}{l}\text { Murawski and } \\
\text { Hamrick (1991) }\end{array}$ \\
\hline $\begin{array}{l}\text { Malvaceae } \\
\text { (Bombacoideae) }\end{array}$ & $\begin{array}{l}\text { Cavanillesia } \\
\text { platanifolia }\end{array}$ & Panama & $\begin{array}{l}\text { Canopy, gap- } \\
\text { specialist, } \\
\text { rare }\end{array}$ & $\begin{array}{l}\text { Hawk moths, } \\
\text { humming birds, } \\
\text { bees }\end{array}$ & Allozymes & $\begin{array}{l}\text { Progeny from a } \\
\text { single population }\end{array}$ & $t_{\mathrm{m}}=0.57$ and 0.35 & & $\begin{array}{l}\text { Murawski et al } \\
\text { (1990) }\end{array}$ \\
\hline $\begin{array}{l}\text { Malvaceae } \\
\text { (Bombacoideae) }\end{array}$ & Ceiba pentandra & $\begin{array}{l}\text { Central } \\
\text { Amazonia }\end{array}$ & $\begin{array}{l}\text { Emergent, } \\
\text { fast-growing }\end{array}$ & Bats & Allozymes & $\begin{array}{l}\text { Progeny from two } \\
\text { locations }\end{array}$ & $\begin{array}{l}91 \text { and } 71 \% \text { seeds } \\
\text { outcrossed }\end{array}$ & & $\begin{array}{l}\text { Gribel et al } \\
\text { (1999) }\end{array}$ \\
\hline $\begin{array}{l}\text { Malvaceae } \\
\text { (Bombacoideae) }\end{array}$ & Ceiba pentandra & $\begin{array}{l}\text { Central } \\
\text { Amazonia }\end{array}$ & $\begin{array}{l}\text { Emergent, } \\
\text { fast-growing }\end{array}$ & Bats & Microsatellites & $\begin{array}{l}\text { Leaves and progeny } \\
\text { from } 400 \mathrm{~km}^{2} \text { forest }\end{array}$ & $\begin{array}{l}24.7 \% \text { selfed seeds at } \\
\text { population level }\end{array}$ & $\begin{array}{l}\text { Several matings } \\
\text { over }>5 \mathrm{~km} \text {; } \\
\text { longest dispersal } \\
18.6 \mathrm{~km}\end{array}$ & $\begin{array}{l}\text { Gribel, reported } \\
\text { in Wilson et al } \\
\text { (2000) }\end{array}$ \\
\hline $\begin{array}{l}\text { Malvaceae } \\
\text { (Bombacoideae) }\end{array}$ & Ceiba pentandra & Costa Rica & $\begin{array}{l}\text { Emergent, } \\
\text { fast-growing }\end{array}$ & Bats & Microsatellites & $\begin{array}{l}\text { Progeny from two } \\
\text { populations }\end{array}$ & $t_{\mathrm{m}}=0.623$ & & Lobo et al (2005) \\
\hline $\begin{array}{l}\text { Malvaceae } \\
\text { (Bombacoideae) }\end{array}$ & Ceiba pentandra & Panama & $\begin{array}{l}\text { Emergent, } \\
\text { fast-growing }\end{array}$ & $\begin{array}{l}\text { Bats, birds, bees, } \\
\text { beetles, wasps, } \\
\text { beetles }\end{array}$ & Allozymes & $\begin{array}{l}\text { Progeny from a } \\
\text { single plot }\end{array}$ & $\begin{array}{l}t_{\mathrm{m}}=0.689 \pm 0.032 ; \text { no } \\
\text { biparental inbreeding }\end{array}$ & & $\begin{array}{l}\text { Murawski and } \\
\text { Hamrick (1991, } \\
\text { 1992a) }\end{array}$ \\
\hline
\end{tabular}


Table 1 Continued

\begin{tabular}{|c|c|c|c|c|c|c|c|c|c|}
\hline Family & Species & Region & Life history & Pollination system & Genetic marker & Sampling design & $\mathrm{t}_{m}$ & Gene flow & Reference \\
\hline $\begin{array}{l}\text { Malvaceae } \\
\text { (Bombacoideae) }\end{array}$ & Pachira quinata & Costa Rica & Canopy & $\begin{array}{l}\text { Bats, sphingid } \\
\text { moths }\end{array}$ & Allozymes & $\begin{array}{l}\text { Progeny from } \\
\text { continuous and } \\
\text { fragmented forest } \\
\text { plots }\end{array}$ & $\begin{array}{l}t_{\mathrm{m}}=0.915 \pm 0.043 \\
\text { (continuous) and } \\
t_{\mathrm{m}}=0.777 \pm 0.114 \\
\text { (isolated trees) }\end{array}$ & & Fuchs et al (2003) \\
\hline $\begin{array}{l}\text { Malvaceae } \\
\text { (Bombacoideae) }\end{array}$ & $\begin{array}{l}\text { Quararibea } \\
\text { asterolepis }\end{array}$ & Panama & $\begin{array}{l}\text { Canopy, slow- } \\
\text { growing, } \\
\text { shade- } \\
\text { tolerant, } \\
\text { common }\end{array}$ & $\begin{array}{l}\text { Hawk moths, } \\
\text { monkeys, bats }\end{array}$ & Allozymes & $\begin{array}{l}\text { Progeny from a } \\
\text { single plot }\end{array}$ & $t_{\mathrm{m}}=1.008 \pm 0.010$ & & $\begin{array}{l}\text { Murawski et al } \\
(1990) ; \\
\text { Murawski and } \\
\text { Hamrick (1991) }\end{array}$ \\
\hline Meliaceae & $\begin{array}{l}\text { Carapa } \\
\text { guianensis }\end{array}$ & Costa Rica & Canopy & $\begin{array}{l}\text { Small insects, } \\
\text { moths }\end{array}$ & Allozymes & $\begin{array}{l}\text { Progeny from two } \\
\text { populations }\end{array}$ & $t_{\mathrm{m}}=0.986$ and 0.967 & & Hall et al (1994) \\
\hline Meliaceae & Carapa procera & French Guiana & Small tree & & Allozymes & $\begin{array}{l}\text { Progeny from plots } \\
\text { within } 300 \text { ha } \\
\text { natural forest }\end{array}$ & $\begin{array}{l}t_{\mathrm{m}}=0.85 \text { for unlogged } \\
\text { plots and } 0.63 \text { for } \\
\text { logged plots }\end{array}$ & & $\begin{array}{l}\text { Doligez and Joly } \\
\text { (1997) }\end{array}$ \\
\hline Meliaceae & Cedrela odorata & Costa Rica & Canopy & $\begin{array}{l}\text { Small insects, } \\
\text { moths }\end{array}$ & Allozymes & $\begin{array}{l}\text { Progeny from a } \\
30 \mathrm{~km} \text { transect of } \\
\text { disturbed forest }\end{array}$ & $t_{\mathrm{m}}=0.969 \pm 0.024$ & & James et al (1998) \\
\hline Meliaceae & Swietenia humilis & Costa Rica & Canopy & $\begin{array}{l}\text { Bees, moths, } \\
\text { thrips }\end{array}$ & Microsatellites & $\begin{array}{l}\text { Progeny from three } \\
\text { forest fragment } \\
\text { plots and one } \\
\text { continuous forest } \\
\text { plot }\end{array}$ & & $\begin{array}{l}\text { Pollen dispersal } \\
\text { up to } 4.5 \mathrm{~km} \\
\text { between forest } \\
\text { fragments }\end{array}$ & White et al (2002) \\
\hline Meliaceae & $\begin{array}{l}\text { Swietenia } \\
\text { macrophylla }\end{array}$ & $\begin{array}{l}\text { Eastern } \\
\text { Amazonia }\end{array}$ & Canopy & $\begin{array}{l}\text { Bees, moths, } \\
\text { thrips }\end{array}$ & Microsatellites & $\begin{array}{l}\text { Leaves and progeny } \\
\text { from } 1 \text { logged } \\
\text { population }\end{array}$ & $t_{\mathrm{m}}=0.958 \pm 0.025$ & & Lemes (2000) \\
\hline Meliaceae & $\begin{array}{l}\text { Swietenia } \\
\text { macrophylla }\end{array}$ & Costa Rica & Canopy & $\begin{array}{l}\text { Bees, moths, } \\
\text { thrips }\end{array}$ & Microsatellites & $\begin{array}{l}\text { Leaves and progeny } \\
\text { from three } \\
\text { populations }\end{array}$ & $\begin{array}{l}50 \text { and } 28.8 \% \text { progeny } \\
\text { outcrossed }\end{array}$ & $\begin{array}{l}\text { Limited pollen } \\
\text { movement }\end{array}$ & Lowe et al (2003) \\
\hline Meliaceae & $\begin{array}{l}\text { Trichilia } \\
\text { tunerculata }\end{array}$ & Panama & Canopy & Possibly bees & Allozymes & $\begin{array}{l}\text { Progeny from a } \\
\text { single plot }\end{array}$ & $t_{\mathrm{m}}=1.077 \pm 0.028$ & & $\begin{array}{l}\text { Murawski and } \\
\text { Hamrick (1991) }\end{array}$ \\
\hline Moraceae & $\begin{array}{l}\text { Brosimum } \\
\text { alicastrum }\end{array}$ & Panama & Canopy & Bees, wind & Allozymes & $\begin{array}{l}\text { Progeny from a } \\
\text { single plot }\end{array}$ & $t_{\mathrm{m}}=0.875 \pm 0.035$ & & $\begin{array}{l}\text { Murawski and } \\
\text { Hamrick (1991) }\end{array}$ \\
\hline Moraceae & $\begin{array}{l}\text { Cecropia } \\
\text { obtusifolia }\end{array}$ & Mexico & $\begin{array}{l}\text { Canopy, } \\
\text { pioneer }\end{array}$ & Wind & Allozymes & $\begin{array}{l}\text { Leaves and progeny } \\
\text { from nine sites }\end{array}$ & $t_{\mathrm{m}}=0.974 \pm 0.024$ & & $\begin{array}{l}\text { Alvarez-Buylla } \\
\text { and Garay } \\
\text { (1994) }\end{array}$ \\
\hline Moraceae & $\begin{array}{l}\text { Ficus citrifolia, } \\
\text { F. dugandii, } \\
\text { F. nymphiifolia, } \\
\text { F. obtusifolia, } \\
\text { F. perforate, } \\
\text { F. pertusa and } \\
\text { F. popenoei }\end{array}$ & Panaa & Canopy & Fig wasps & Allozymes & $\begin{array}{l}\text { Leaves and progeny } \\
\text { from an area of } \\
\text { approximately } 15 \mathrm{~km}^{2}\end{array}$ & & $\begin{array}{l}\text { Extensive pollen } \\
\text { movement over } \\
5.8-14.2 \mathrm{~km} \text {; } \\
\text { breeding units } \\
\text { between } 106 \\
\text { and } 632 \mathrm{~km}^{2}\end{array}$ & $\begin{array}{l}\text { Nason et al } \\
(1998)\end{array}$ \\
\hline Moraceae & Sorocea affinis & Panama & Understory & $\begin{array}{l}\text { Small bees, } \\
\text { wind }\end{array}$ & Allozymes & $\begin{array}{l}\text { Progeny from a } \\
\text { single plot }\end{array}$ & $\begin{array}{l}t_{\mathrm{m}}=1.089 \pm 0.045 \\
\text { and } 0.969 \pm 0.020\end{array}$ & & $\begin{array}{l}\text { Murawski and } \\
\text { Hamrick (1991) }\end{array}$ \\
\hline Rubiaceae & $\begin{array}{l}\text { Psychotria } \\
\text { faxlucens }\end{array}$ & Mexico & Understory & Moths & Allozymes & $\begin{array}{l}\text { Progeny from two } \\
25 \times 30 \mathrm{~m}^{2} \text { plots in } \\
700 \text { ha forest }\end{array}$ & $t_{\mathrm{m}}=0.898$ and 0.685 & & $\begin{array}{l}\text { Pérez-Nasser } \\
\text { et al (1993) }\end{array}$ \\
\hline Staphyleaceae & $\begin{array}{l}\text { Turpinia } \\
\text { occidentalis }\end{array}$ & Panama & Subcanopy & $\begin{array}{l}\text { Variety of small } \\
\text { insects }\end{array}$ & Allozymes & $\begin{array}{l}\text { Leaves and progeny } \\
\text { from a } 50 \text { ha } \\
\text { population }\end{array}$ & $t_{\mathrm{m}}=1.006 \pm 0.090$ & $\begin{array}{l}\text { Small fraction } \\
\text { of matings over } \\
\text { long distances }\end{array}$ & Stacy et al (1996) \\
\hline
\end{tabular}


play predominant outcrossing or mixed mating, African populations are reportedly completely self-compatible (Baker, 1955, 1965), which may have been a requisite for establishing founder populations following oceanic dispersal. At the landscape scale, Ribeiro and Lovato (2004) found two populations of S. multijuga (Fabaceae) to have significantly different outcrossing rates $\left(t_{\mathrm{m}}=\right.$ 0.838 and 0.540 ), thought to be a reflection of genetic and environmental differences among populations. Murawski and Hamrick (1992b) attributed differences in the outcrossing rate of two populations of C. platanifolia to differences in flowering-plant densities. Mating system variation at the regional scale may also reflect differences among populations in the genetic load that leads to inbreeding depression.

Changes in the landscape, such as selective logging, deforestation and habitat destruction alter population density, demographic structure, phenology and the abundance, diversity and behaviour of pollinator communities (Dick, 2001; Lowe et al, 2005), thereby impinging on the mating system. Doligez and Joly (1997) found significant differences between outcrossing rates of Carapa procera in undisturbed plots and logged plots $\left(t_{\mathrm{m}}=0.85\right.$ and 0.63 , respectively). A significant increase in self-fertlization rates has been reported for the emergent legume $D$. excelsa in undisturbed and fragmented forests in Brazil $\left(t_{\mathrm{m}}=0.897\right.$ and 0.845, respectively) (Dick et al, 2003), and in Costa Rica reduced outcrossing rates were documented for Symphonia globulifera $\left(t_{\mathrm{m}}=0.902\right.$ and 0.739; Aldrich and Hamrick, 1998) and P. quinata $\left(t_{\mathrm{m}}=0.915\right.$ and 0.777; Fuchs et al, 2003) in disturbed habitats that contained low population densities of reproductive trees.

Other factors are expected to cause variation in mating system, but have not been covered here due to lack of comparative data. These factors include the influence of pollination syndrome (Barrett, 2003), canopy height (understory, canopy, emergent) and colonisation guild. The pollination syndromes of neotropical trees are highly variable, and include small and large insects (eg Bawa et al, 1985), hummingbirds (the principal avian pollinator in the neotropics; eg Degen et al, 2004), bats (eg Murawski and Hamrick, 1991; Collevatti et al, 2001) and, rarely, wind (eg Alvarez-Buylla and Garay, 1994) or wind-directed insect pollination (Nason et al, 1998). Insect pollination syndromes are well represented, but important and distinctive groups have been overlooked, such as the small beetle pollination system of the Myristicaceae (Armstrong and Irvine, 1989).

\section{Pollen dispersal}

The first allozyme studies of pollen dispersal in tropical trees tracked rare alleles or used fractional paternity analysis, permitting direct inference of pollen dispersal over hundreds of metres in undisturbed forest (Hamrick and Murawski, 1990; Boshier et al, 1995b; Loveless et al, 1998). Hamrick and Murawski (1990) found that $20 \%$ of pollen moved over $750 \mathrm{~m}$ in P. elegans, and over $25 \%$ of pollen moved more than $500 \mathrm{~m}$ in Tachigali versicolor in undisturbed forest on Barro Colorado Island (BCI) Panama (also see Loveless et al, 1998). Both tree species are large, mass-flowering papilionoid legumes found in low densities, and are pollinated by large bees (eg Centris, Xylocopa). Stacy et al (1996) studied pollen dispersal in three tree species found in low densities ( $\sim 0.3$ tree/ha) on BCI, whose small-insect floral visitors include beetles, small bees and moths. The combination of rarity and small-insect pollination leads to an expectation of low outcrossing and nearest-neighbour mating (Levin and Kerster, 1974). Counter to this expectation, all three populations were $100 \%$ outcrossed, and pollen dispersal distances exceeded the mean distances to the nearest potential mates. Violation of nearest-neighbour mating may occur in low-density populations because nearest flowering neighbours are in fact not close $(>50 \mathrm{~m})$ and small insects may frequently not fly in the direction of the nearest neighbour. In a study of an almost monospecific stand of moth-pollinated Cordia alliadora (Boraginaceae) in Costa Rica, Boshier et al (1995b) reported a majority of pollen dispersal at $75 \mathrm{~m}$, with some travelling over $280 \mathrm{~m}$. Though the number of comparable studies is low, these results suggest that trees in low-density populations receive pollen over larger distances than do densely spaced trees.

In addition, or possibly due to an ecological or pollination byproduct of density, the relative canopy position and colonization guild of tropical trees are also expected to influence gene flow and mating system dynamics (Bawa et al, 1985; Nason and Hamrick, 1997). However, few mating system studies have been conducted on subcanopy/understory species or slow-growing, shade-tolerant species, making comparisons with the better-studied classes of canopy/emergents and pioneer species, respectively, problematic. Such studies would have to involve a large sample of species with sufficient representation of other key traits (ie inbreeding depression, population density and phylogeny), and may be possible in future, following further work on individual case studies.

Fig trees (Ficus) on BCI are the lowest density populations yet studied with respect to pollen dispersal (Nason et al, 1996, 1998). Figs are generally obligately outcrossing and are pollinated by miniscule $(\sim 2 \mathrm{~mm})$ wasps (Agaonidae), which live only for 2-3 days. Figs usually have species-specific relationship with wasp pollinators (for counterexamples, see Molbo et al, 2003). Several species of Ficus on BCI occur at very low population densities; for example, there are only 20 known adults of Ficus dugandii in $16 \mathrm{~km}^{2}$ of forest of BCI. Often, a single pollinating wasp visits the inverted fig inflorescence, in which case the seeds from that fruit are full siblings. Nason et al (1998) reconstructed paternal diploid genotypes of singly pollinated fruits through exclusion analysis, and found that fruits on maternal trees had numerous pollen donors. In all, 15 fruits from a F. dugandii, for example, yielded a minimum estimate of 11 pollen donors. The authors suggest that the number of pollen donors would have continued to rise if more single-sired fruits had been genotyped. Based on the population density, the authors estimated that pollen is routinely dispersed over distances of $5.8-14.2 \mathrm{~km}$, and that the breeding areas of these figs range from 106 to $632 \mathrm{~km}^{2}$. How do tiny, ephemeral wasps orient themselves and travel such long distances between conspecific trees? The authors suggest that the fig wasps become windborne, then hone in on scents produced by the receptive flowering tree. Further research is needed to precisely characterize mating patterns in this 
fascinating system, and to test the hypothesis of directed pollen dispersal.

Vertebrate pollinators are also capable of maintaining tree reproductive populations over large spatial scales. For example, bats have been noted to carry $C$. pentandra pollen over distances greater than $5 \mathrm{~km}$ in many instances, and a maximum dispersal distance of $18.6 \mathrm{~km}$ observed (Gribel, reported in Wilson et al, 2001), the greatest single pollen dispersal distance so far recorded in the literature.

The development of analytical techniques such as Two-Gener (Austerlitz et al, 2004; Smouse and Sork, 2004) has made it possible to infer the shape of the pollen dispersal curve using genotyped seed arrays, mapped maternal trees and an estimate of population density. The method treats the pollen pool of a progeny array as a population, then estimates genetic differentiation with an $F_{\text {st }}$-based statistic. Using this approach, Dick et al (2003) estimated the mean pollen dispersal distance of Dinzia excelsa of $212 \mathrm{~m}$ in undisturbed forest. Degen et al (2004) estimate the mean pollen dispersal distances for $S$. globulifera of between 27 and $53 \mathrm{~m}$, depending on the model used. The shorter pollen dispersal distance may be explained by the pollination system of $S$. globulifera (hummingbird) compared to D. excelsa (small bee), or by the difference in population density: the effective density of $S$. globulifera is more than 10 times greater than D. excelsa.

\section{Conclusions and future recommendations}

Neotropical tree species exhibit characteristics in the reproductive system that allow demographic persistence and heterozygosity even at low population densities. Selection pressures against inbreeding in low-density populations of tropical trees has presumably been the main driving force responsible for the evolution of these characters, which include (1) the constellation of attraction mechanisms for animal pollination and pollinator constancy (see Endress, 1998), and (2) the widespread occurrence of self-incompatibility mechanisms (eg Bawa et al, 1985). Genetic studies show that tropical animals can be very efficient vectors of pollen flow, capable of promoting outcrossing and successfully moving pollen between distantly spaced individuals in heterogeneous habitats.

Despite these advances in our understanding of reproduction and gene flow in neotropical trees, the available research barely touches the surface of the taxonomic and ecological diversity represented in these plants. Several areas for future research can be highlighted based on the findings of this review:

(1) Further work is required to examine the fitness consequences of mating system variation. This requires focus on the effects of inbreeding depression, and measurement of the relative fitness of selfed vs outcrossed progeny at various life-history stages.

(2) Researchers need to be aware of the possibility of apomixes in the seed production. This will be evident with microsatellites when maternal trees and seeds share identical multilocus genotypes.

(3) There is a need to understand how particular classes of pollinators serve as pollen vectors. These include the small-beetle pollination system found in the nutmeg family (Myristicaceae), euglossine bee and hummingbird pollination.

(4) Genetic studies of pollen dispersal often fail to document the putative pollinators. While many neotropical trees are visited by generalist pollinators, there is often a narrower range of effective pollinators (Bawa, 1992) and pollinator communities may shift across habitats (Dick, 2001). The effective pollinators need to be identified so that generalizations can be applied to other plant taxa sharing those pollinators.

(5) More studies should document the range of mating system variation across multiple seasons, as significant seasonal variation has been noted where investigated (eg Rocha and Aguilar, 2001).

(6) More work is required to examine the relationship between mating system and key ecosystem class (canopy vs understory, pioneers us shade tolerant). Several theoretical predictions have been made connected with population density and pollinator motility, but lack of comparative data sets has so far not allowed rigorous empirical assessment.

\section{References}

Aldrich PR, Hamrick JL (1998). Reproductive dominance of pasture trees in a fragmented tropical forest mosaic. Science 281: 103-105.

Alvarez-Buylla ER, Garay AA (1994). Population genetic structure of Cecropia obtusifolia, a tropical pioneer tree species. Evolution 48: 437-453.

Armstrong JE, Irvine AK (1989). Floral biology of Myristica insipida (Myristicaceae), a distinctive beetle pollination system. Am J Bot 76: 86-94.

Austerlitz F, Dick C, Dutech C, Klein EK, Oddou-Muratorio S, Smouse PE et al (2004). Using genetic markers to estimate the pollen dispersal curve. Mol Ecol 13: 937-954.

Baker HG (1955). Self compatibility and establishment after long-distance dispersal. Evolution 9: 347-349.

Baker HG (1959). Reproductive methods as factors in speciation in flowering plants. Cold Spring Harb Symp Quant Biol 24: 177-199.

Baker HG (1960). Apomixis and polyembryony in Pachira oleaginea (Bombacaceae). Am J Bot 47: 296-302.

Baker HG (1965). The evolution of the cultivated kapok tree: a probable West African product. In: Brokensha (ed) Ecology and Economic Development in Tropical Africa. Research Series No. 9, Institute of International Studies, University of California: Berkeley, CA, USA. pp 185-216.

Barrett SCH (2003). Mating strategies in flowering plants: the outcrossing-selfing paradigm and beyond. Philos Trans $R$ Soc Lond Ser B 358: 991-1004.

Bawa KS (1992). Mating systems, genetic differentiation and speciation in tropical rainforest plants. Biotropica 24: 250-255.

Bawa KS, Perry DR, Beach JH (1985). Reproductive biology of tropical lowland rainforest trees. 1. Sexual systems and incompatibility mechanisms. Am J Bot 72: 331-345.

Bittencourt NS, Semir J (2005). Late-acting self-incompatibility and other breeding systems in Tabebuia (bignoniaceae). Int $J$ Plant Sci 166: 493-506.

Boshier DH, Chase MR, Bawa KS (1995a). Population genetics of Cordia alliodora (Boraginaceae), a neotropical tree. 2. Mating system. Am J Bot 82: 476-483.

Boshier DH, Chase MR, Bawa KS (1995b). Population genetics of Cordia alliodora (Boraginaceae), a neotropical tree. 3. Gene flow, neighborhood, and population substructure. Am J Bot 82: 484-490. 
Collevatti RG, Grattapaglia D, Hay JD (2001). High resolution microsatellite based analysis of the mating system allows the detection of significant biparental inbreeding in Caryocar brasiliense, an endangered tropical tree species. Heredity 86: 60-67.

Corner EJH (1954). The evolution of tropical forest. In: Huxley JS, Hardy AC, Ford EB (eds) Evolution as a Process. Allen \& Unwin: London. pp 34-46.

Dawson IK, Waugh R, Simons AJ, Powell W (1997). Simple sequence repeats provide a direct estimate of pollenmediated gene dispersal in tropical tree Gliricidia sepium. Mol Ecol 6: 179-183.

Degen B, Bandou E, Caron H (2004). Limited pollen dispersal and biparental inbreeding in Symphonia globulifera in French Guiana. Heredity 93: 585-591.

Dick CW (2001). Genetic rescue of remnant tropical trees by an alien pollinator. Proc Roy Soc Lond B 268: 2391-2396.

Dick CW, Etchelecu G, Austerlitz F (2003). Pollen dispersal of tropical trees (Dinizia excelsa: Fabaceae) by native insects and African honeybees in pristine and fragmented Amazonian rainforest. Mol Ecol 12: 753-764.

Doligez A, Joly HI (1997). Mating system of Carapa procera in the French Guiana tropical rainforest. Am J Bot 84: 461-470.

Duncan EJ (1970). Ovule and embryo ontogenesis in Bombacopsis glabra (Pasq.) A. Robins. Ann Bot 34: 32-46.

Dutech C, Seiter J, Petronelli P, Joly HI, Jarne P (2002). Evidence of low gene flow in a neotropical clustered tree species in two rainforest stands of French Guiana. Mol Ecol 11: $725-738$.

Eguiarte LE, Perez-Nasser N, Pinero D (1992). Genetic structure, outcrossing rate and heterosis in Astrocaryum mexicanum (tropical palm): implications for evolution and conservation. Heredity 69: 217-228.

Endress PK (1998). Diversity and Evolutionary Biology of Tropical Flowers, 2nd edn. Cambridge University Press: Cambridge, UK. p 511

Fedorov AA (1966). The structure of the tropical rain forest and speciation in the humid tropics. J Ecol 54: 1-11.

Franceschinelli EV, Bawa KS (2000). The effect of ecological factors on the mating system of a South American shrub species (Helicteres brevispira). Heredity 84: 116-123.

Fuchs EJ, Lobo JA, Quesada M (2003). Effects of forest fragmentation and flowering phenology on the reproductive success and mating patterns of the tropical dry forest tree Pachira quinata. Cons Biol 17: 149-157.

Gaiotto FA, Grattapaglia D, Vencovsky R (2003). Genetic structure, mating system, and long-distance gene flow in heart of palm (Euterpe edulis Mart.). J Hered 94: 399-406.

Gibson JP, Wheelwright NT (1996). Mating system dynamics of Ocotea tenera (Lauraceae), a gynodioecious tropical tree. Am J Bot 83: 890-894.

Gribel R, Gibbs PE (2002). High outbreeding as a consequence of selfed ovule mortality and single vector bat pollination in the Amazonian tree Pseudobombax munguba (Bombacaceae). Int J Plant Sci 163: 1035-1043.

Gribel R, Gibbs PE, Queiróz AL (1999). Flowering phenology and pollination biology of Ceiba pentandra (Bombacaceae) in Central Amazonia. J Trop Ecol 15: 247-263.

Hall P, Orrell LC, Bawa KS (1994). Genetic diversity and mating system in a tropical tree, Carapa guianensis (Meliaceae). Am J Bot 81: 1104-1111.

Hall P, Walker S, Bawa K (1996). Effect of forest fragmentation on genetic diversity and mating system in a tropical tree, Pithecellobium elegans. Cons Biol 10: 757-768.

Hamrick JL, Murawski DA (1990). The breeding structure of tropical tree populations. Pl Sp Biol 5: 157-165.

Hubbell SP, Foster RB (1983). Diversity of canopy trees in a neotropical forest and implications for conservation. In Sutton SL, Whitmore TC, Chadwick AC (eds) Tropical Rain Forest: Ecology and Management. Blackwell Scientific Publications: Oxford. pp 25-41.
Hufford KM, Hamrick JL (2003). Viability selection at three early life stages of the tropical tree, Platypodium elegans (Fabaceae, Papilionoideae). Evolution 57: 518-526.

James T, Vege S, Aldrich P, Hamrick JL (1998). Mating systems of three tropical dry forest tree species. Biotropica 30: 587-594.

Kaur A, Ha CO, Jong K, Sands VE, Chan HT, Soepadmo E et al (1978). Apomixis may be widespread among trees of the climax rain forest. Nature 271: 440-443.

Latouche-Hallé C, Ramboer A, Bandou E, Caron H, Kremer A (2004). Long-distance pollen flow and tolerance to selfing in a neotropical tree species. Mol Ecol 13: 1055-1064.

Lemes MR (2000). Population genetic structure and mating system of Swietenia macrophylla (Meliaceae) in the Brazilian Amazon: implications for Conservation. PhD Thesis. University of Stirling: Stirling, UK

Levin DA, Kerster HW (1974). Gene flow in seed plants. Evol Biol 7: 139-220.

Lobo JA, Quesada M, Stoner KE (2005). Effects of pollination by bats on the mating system of Ceiba pentandra (Bombacaceae) populations in two tropical life zones in Costa Rica. Am J Bot 92: $370-376$.

Loveless MD, Hamrick JL, Foster RB (1998). Population structure and mating system in Tachigali versicolor, a monocarpic neotropical tree. Heredity 81: 134-143.

Lowe AJ, Boshier D, Ward M, Bacles CFE, Navarro C (2005). Genetic resource impacts of habitat loss and degradation; reconciling empirical evidence and predicted theory for neotropical trees. Heredity 95: 255-273.

Lowe AJ, Jourde B, Breyne P, Colpaert N, Navarro C, Wilson J et al (2003). Fine-scale genetic structure and gene flow within Costa Rican populations of mahogany (Swietenia macrophylla). Heredity 90: 268-275.

Molbo D, Machado C, Sevenster JG, Keller L, Herre EA (2003). Cryptic species of fig-pollinating wasps: implications for the evolution of the fig-wasp mutualism, sex allocation and precision of adaptation. Proc Natl Acad Sci USA 100: 5867-5872.

Murawski DA, Bawa KS (1994). Genetic structure and mating system of Stemonoporus oblongifolius (Dipterocarpaceae) in Sri Lanka. Am J Bot 81: 155-160.

Murawski DA, Dayanandan B, Bawa KS (1994). Outcrossing rates of two endemic Shorea species from Sri Lanka tropical rain forests. Biotropica 26: 23-29.

Murawski DA, Hamrick JL (1991). The effect of the density of flowering individuals on the mating systems of nine tropical tree species. Heredity 67: 167-174.

Murawski DA, Hamrick JL (1992a). Mating system and phenology of Ceiba pentandra (Bombacaceae) in Central Panama. I Hered 83: 401-404.

Murawski DA, Hamrick JL (1992b). The mating system of Cavanillesia platanifolia under extremes of flowering-tree density: a test of predictions. Biotropica 24: 99-101.

Murawski DA, Hamrick JL, Hubbell SP, Foster RB (1990). Mating systems of two Bombacaceous trees of a neotropical moist forest. Oecologia 82: 501-506.

Nason JD, Hamrick JL (1997). Reproductive and genetic consequences of forest fragmentation: two case studies of neotropical canopy trees. I Hered 88: 264-276.

Nason JD, Herre EA, Hamrick JL (1996). Paternity analysis of the breeding structure of strangler fig populations: evidence for substantial long-distance wasp dispersal. J Biogeogr 23: 501-512.

Nason JD, Herre EA, Hamrick JL (1998). The breeding structure of a tropical keystone plant resource. Nature 391: 685-687.

Neal PR, Anderson GJ (2005). Are 'mating systems' 'breeding systems' of inconsistent and confusing terminology in plant reproductive biology? Or is it the other way around? Plant Syst Evol 250: 173-185.

Oliveira PE, Gibbs PE, Barbosa AA, Talavera S (1992). Contrasting breeding systems in two Eriotheca (Bombaca- 
ceae) species of the Brazilian cerrados. Plant Syst Evol 179: 207-219.

O'Malley DM, Bawa KS (1987). Mating system of a tropical rainforest tree species. Am J Bot 74: 1143-1149.

O’Malley DM, Buckley DP, Prance GT, Bawa KS (1988). Genetics of Brazil nut (Bertholletia excelsa Humb. \& Bonpl.: Lecythidaceae). 2. Mating system. Theor Appl Genet 76: 929-932.

Pennington RT, Dick CW (2004). The role of immigrants in the assembly of the South American rainforest tree flora. Philos Trans $R$ Soc Lond Ser B 359: 1611-1622.

Pérez-Nasser N, Eguiarte LE, Pinero D (1993). Mating system and genetic-structure of the distylous tropical tree Psychotria faxlucens (Rubiaceae). Am J Bot 80: 45-52.

Ribeiro RA, Lovato MB (2004). Mating system in a neotropical tree species, Senna multijuga (Fabaceae). Genet Mol Biol 27: 418-424.

Ritland K (1986). Joint maximum likelihood estimation of genetic and mating structure using open-pollinated progenies. Biometrics 42: 25-43.

Ritland K, Jain S (1981). A model for the estimation of outcrossing rate and gene frequencies using $n$ independent loci. Heredity 47: 35-52.
Rocha OJ, Aguilar G (2001). Variation in the breeding behavior of the dry forest tree Enterolobium cyclocarpum (Guanacaste) in Costa Rica. Am J Bot 88: 1600-1606.

Seavey SR, Bawa KS (1986). Late-acting self-incompatibility in angiosperms. Bot Rev 52: 195-219.

Smouse PE, Sork VL (2004). Measuring pollen flow in forest trees: an exposition of alternative approaches. Forest Ecol Manage 197: 21-38.

Stacy EA, Hamrick JL, Nason JD, Hubbell SP, Foster RB, Condit R (1996). Pollen dispersal in low-density populations of three neotropical tree species. Am Nat 148: 275-298.

Thomas SC (1997). Geographic parthenogenesis in a tropical forest tree. Am J Bot 84: 1012-1015.

White GM, Boshier DH, Powell W (2002). Increased pollen flow counteracts fragmentation in a tropical dry forest: an example from Swietenia humilis Zuccarini. Proc Natl Acad Sci USA 99: 2038-2042.

Wilson J, Lowe AJ, Cavers S, Navarro C, Hernandez M, Kremer A et al (2001). Assessment of levels and dynamics of intraspecific genetic diversity of tropical trees. Final Scientific Report 1997-2001. European Commission. 76pp. 